\title{
A dextran-based warming method for preparing leukocyte-rich plasma and its clinical application for endotoxin assay
}

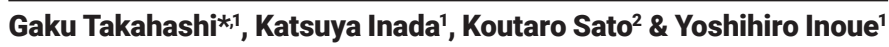

\section{ABSTRACT}

We devised a method using dextran for obtaining leukocyte-rich plasma (LRP) to measure endotoxin in blood. In order to find the optimal temperature for obtaining LRP, the measurement results were examined using samples prepared at 37 and $0^{\circ} \mathrm{C}$. Sample separation time of LRP was significantly shorter at $37^{\circ} \mathrm{C}$ than at $0^{\circ} \mathrm{C}$. Endotoxin measurement values showed a strong correlation between the two groups but many of the LRPs made at $37^{\circ} \mathrm{C}$ had measurements above those of the LRPs prepared at $0^{\circ} \mathrm{C}$. The diagnostic accuracy for gram-negative bacterial infection was superior for LRP prepared at $37^{\circ} \mathrm{C}$, with sensitivity and specificity of 96.8 and $100 \%$, respectively.

\section{METHOD SUMMARY}

Plasma is usually used for measurement of blood endotoxin. Here, leukocyte-rich plasma (LRP) was obtained using an erythrocyte aggregation agent, dextran, and used for the assay. It was found that when LRP was separated at $37^{\circ} \mathrm{C}$ (referred to here as the LRP37 method), endotoxin values were higher than those obtained at $0^{\circ} \mathrm{C}$ (LRPO method). The sensitivity and specificity of the LRP37 method were superior to those achieved using the LRP0 method.

\section{KEYWORDS}

dextran • endotoxin • endotoxin-specific limulus amebocyte lysate assay - leukocyte-rich plasma • platelet-rich plasma $\cdot$ polymyxin $B \cdot$ sepsis $\cdot$ turbidimetric kinetic assay

${ }^{1}$ Department of Critical Care Medicine, Iwate Medical University, Morioka, Japan; ${ }^{2}$ Department of Orthopedic surgery, Iwate Medical University, Morioka, Japan; *Author for correspondence: gakut@iwate-med.ac.jp

BioTechniques 68: 300-304 (June 2020) 10.2144/btn-2020-0005
Endotoxins are cell wall components of gram-negative bacteria with various biological activities. In patients with bacterial infections, sepsis is typically diagnosed by measuring blood endotoxin concentrations after exposing samples to blood cell extracts from the horseshoe crab. In Japan, the standard practice for measuring blood endotoxin concentrations is an endotoxin-specific limulus amebocyte lysate ( $L A L)$ assay using platelet-rich plasma (PRP). However, the sensitivity of this assay is too low to be considered diagnostically efficient [1].

Endotoxins bind to leukocytes through TLR4 [2] or CD18/CD11b [3], after which the endotoxins are engulfed into the phagocytic cells [4]. It follows that endotoxin levels should be measured on the surface as well as inside the cells during $L A L$ assays. We first attempted to use the leukocyte fraction (buffy coat) from centrifuged patient blood for the assay [5] and found that the leukocyte fractions had higher endotoxin levels than the plasma fractions in some patients. However, this method is too complicated and impractical for routine use. Therefore, we attempted to use leukocyte-rich plasma (LRP) for the endotoxin assay [1,6] and found once again that the measurement of leukocyte-bound endotoxins was necessary. Separation of LRP was previously performed by adding the hemagglutinating agent hydroxyethyl starch (HES) to the blood [7,8]. However, it has become difficult to obtain HES in recent years; thus, we attempted to determine whether separation with dextran is possible. It has been reported that ice-cold conditions are important for restoring sufficient endotoxin activity (compared with incubation at $37^{\circ} \mathrm{C}$ and based on lipopolysaccharide (LPS) spike experiments on plasma from sepsis patients) [9]. Therefore, in this study, we also investigated whether the measurement accuracy of endotoxins is sufficiently high under ice-cold conditions.

\section{MATERIALS \& METHODS \\ Reagents}

- Dextran T-500 (Pharmacosmo, Holbaek, Denmark), with an average molecular weight of $500,000 \mathrm{Da}$, was dissolved in physiological saline $(6 \% \mathrm{w} / \mathrm{v})$ and autoclaved at $121^{\circ} \mathrm{C}$ for $90 \mathrm{~min}$ to ensure the endotoxin level was below the detection limit $(\varangle 0.04 \mathrm{pg} / \mathrm{ml})$ of the limulus test (endotoxin-free);

- Phosphate-buffered saline (-): The compound powder (Nissui Pharmaceutical, Tokyo) was dissolved in water to a concentration of $0.01 \mathrm{M}$ and autoclaved at $121^{\circ} \mathrm{C}$ for $90 \mathrm{~min}$ to ensure it was endotoxin-free. The solution does not contain calcium or magnesium;

- LPS: LPS from Escherichia coli 0111: B4 (Sigma-Aldrich, MO, USA) was suspended in phosphate-buffered saline $(-)$ at a concentration of $1 \mathrm{mg} /$ $\mathrm{ml}$. The LPS suspension was dispersed using an ultrasonic generator (UR 21-P, Tommy Seiko, Tokyo), dispensed in small volumes, and stored at $-80^{\circ} \mathrm{C}$ until use;

- Plasma pretreatment solution and LAL reagent: a plasma pretreatment solution (0.02\% Triton X-100 Solution, FUJIFILM Wako Pure Chemical Corporation, Osaka, Japan) and Endotoxin Single Test Wako® (Lyophilized product, FUJIFILM Wako Pure Chemical Corporation) were used. Endotoxin Single Test Wako is a reagent specific for endotoxins [10,11];

- Polymyxin B was purchased from Sigma-Aldrich.

\section{Patients}

The total number of patients was 67 ; the patient backgrounds are presented in Table 1. Of these, 47 patients were suspected of sepsis or infection, and no infection was found in the other 20 patients. Among those suspected of sepsis or infection, bacteria were detected in cultures of blood or urine, sputum, ascitic fluid, spinal fluid or wound 
for 45 patients. Gram-negative bacteria were detected in 33 cases, and gram-positive bacteria were detected in 12 cases. Informed consent was obtained from all participants.

\section{Preparation of PRP}

As soon as the blood sample was drawn with heparin, the tube was placed in crushed ice to equilibrate it at $0^{\circ} \mathrm{C}$ (within 5-10 min). To obtain PRP, the blood samples were centrifuged at $1200 \mathrm{~g}$ (3000 r.p.m.) for $40 \mathrm{~s}$ [12]. The endotoxin assay using PRP is hereinafter referred to as the plasma method.

\section{Preparation of LRP}

LRP was prepared under two conditions: at $37^{\circ} \mathrm{C}$ and on ice. A dextran solution (6\%) was equilibrated to the experimental temperature in advance. Immediately after blood collection, the heparinized sample was transported under ambient temperature to our endotoxin research laboratory and equilibrated for 5-10 min at the experimental temperature. Thereafter, the LRP was collected according to the following protocol. Briefly, $800 \mu$ of the equilibrated blood was added to $400 \mu$ l of $6 \%$ dextran T-500, which was also equilibrated to the same temperature, and the mixture was incubated. The supernatant layer separated from the sedimented erythrocytes after 30 min was designated as the LRP. The methods used to prepare the LRP under ice-cooling and at $37^{\circ} \mathrm{C}$ are referred to as the LRPO and LRP37 methods, respectively.
Endotoxin measurement by turbidimetric kinetic assay

PRP or LRP $(100 \mu \mathrm{l})$ obtained using the LRP37 or LRP0 method was added to $900 \mu \mathrm{l}$ of plasma pretreatment solution (i.e., a 10x dilution) and mixed in a vortex mixer (MS-3, IKA Japan, Osaka, Japan). The diluted LRP was mixed five-times in a vortex mixer at 3000 r.p.m. for $10 \mathrm{~s}$ to ensure the destruction of white blood cells. The mixture was heated at $70^{\circ} \mathrm{C}$ for $10 \mathrm{~min}$ and then cooled in iced water for $5 \mathrm{~min}$. The Endotoxin Single Test Wako reagent was dissolved with $200 \mu$ l of the pretreated specimen. The Toxinometer MT-5500 ${ }^{\circledR}$ (Wako Pure Chemical Corporation) was used to obtain measurements. The time taken for the amount of transmitted light to be reduced by $8 \%$ was defined as the gelation time. The endotoxin concentration was calculated using a calibration curve according to the gelation time for standard LPS (E. coli 0111: B4, Wako Pure Chemical Corporation) $[10,11]$. The detection limit for this assay was approximately $0.05 \mathrm{pg} / \mathrm{ml}$; this value was calculated at a maximum set time of $200 \mathrm{~min}$ (this value varied depending on the lot number for the $L A L$ ). Since the plasma was diluted to tenfold, the detection limit for endotoxins was approximately $0.5 \mathrm{pg} / \mathrm{ml}$. Average values were obtained by duplicate measurements of each specimen.

\section{Table 1. Patient characteristics.}

\begin{tabular}{|l|l|l|}
\hline Diagnosis & Noninfection (n) & Infection or sepsis (n) \\
\hline Respiratory disease & 0 & 16 \\
\hline Peritonitis & 0 & 12 \\
\hline Streptococcal toxic shock syndrome & 0 & 6 \\
\hline Bacterial meningitis & 0 & 4 \\
\hline Burns & 3 & 7 \\
\hline Liver abscess & 0 & 1 \\
\hline Tetanus & 0 & 1 \\
\hline Trauma & 9 & 1 \\
\hline Acute pancreatitis & 3 & 0 \\
\hline Malignant hyperthermia & 2 & 0 \\
\hline Healthy & 3 & 0 \\
\hline Total (n = 67) & 20 & 47 \\
\hline
\end{tabular}

\section{Correction of endotoxin content of LRP considering hematocrit value}

Since half the volume of dextran was added to one volume of blood to obtain the LRP, the LRP endotoxin content was corrected using the following formula. The LRP endotoxin value $(\mathrm{pg} / \mathrm{ml})$ was obtained by multiplying the actual value obtained with the LRP method by the coefficient ([A + dextran amount] $/ A$ ), wherein $A=$ blood volume-blood volume $\times$ hematocrit value/100. For example, if the blood volume and hematocrit value are $800 \mathrm{ml}$ and 30 , respectively, $A$ is calculated as $560(800-800 \times 0.3)$. If the dextran volume is $400 \mathrm{ml}$, a multiplying factor of 1.71 is obtained by $(560+400) / 560$. Since the endotoxin levels of LRP and PRP were diluted tenfold in the treatment stage, they were finally multiplied by 10 .

\section{Statistical analysis}

Student's $t$-test, Mann-Whitney $U$ test, Wilcoxon test, creation of receiver operating characteristic (ROC) curves and calculation of specificity and sensitivity were performed using SPSS software. Significant differences were regarded as those with $p$-values of less than 0.05 .

\section{RESULTS \& DISCUSSION}

\section{Incubation conditions for LRP}

\section{preparation}

First, we performed an experiment to obtain LRP under ice-cooled conditions. For this experiment, room temperature was set as the control condition because a previous study reported that endotoxin inactivation in patient plasma occurred at $37^{\circ} \mathrm{C}$ [9]. However, endotoxin levels in the LRP prepared at room temperature tended to be higher than those in the LRP prepared under ice-cooled conditions, contrary to our expectations (data not presented). Therefore, we set the preparation temperature for LRP as $37^{\circ} \mathrm{C}$ (LRP37 method) and compared the endotoxin levels with the values obtained under ice-cooled conditions (LRP0 method). The times to obtain the LRP were $9 \mathrm{~min}$ and $12 \mathrm{~s}$ with the LRP37 method and $12 \mathrm{~min}$ and $34 \mathrm{~s}$ with the LRPO method (no infection group, $n=20, p<0.001$, $t$-test). The results for the 67 samples measured indicated that both values were significantly correlated $\left(R^{2}=0.97\right)$. As shown in Figure 1, the values obtained using the LRP37 method were higher than those obtained using the LRPO 
method in more than $80 \%$ of the cases and especially in cases where gram-negative bacteria were detected, and the average value also tended to be higher (LRPO method: $31.42 \pm 14.12 \mathrm{pg} / \mathrm{ml}$, LRP37 method: $41.46 \pm 14.12 \mathrm{pg} / \mathrm{ml}$ ).

\section{Effect of dextran}

The limulus activity of endotoxin in LPS-spiked physiological saline and in endotoxemic patient plasma was observed after incubation in the presence of dextran for $15 \mathrm{~min}$ at $0^{\circ} \mathrm{C}$ (ice-cooled) or $37^{\circ} \mathrm{C}$ (Figure 2). The limulus activity of LPS appeared to be enhanced depending on the concentration of dextran, especially at $37^{\circ} \mathrm{C}$, in both LPS-spiked physiological saline and endotoxemic patient plasma. This enhancement was completely inhibited in LPS-spiked saline by polymyxin B, confirming that the reactions were not false positives. In addition, when the dextran concentration was set to $6 \%$ or higher, the viscosity of the specimen increased, and accurate measurement could not be performed. Therefore, in this study, the dextran concentration was set to $6 \%$.

\section{Examination using patient samples}

Three methods were compared: the LRP0, LRP37 and plasma methods. The measured values were significantly higher for gramnegative bacteria with all methods. The measured values tended to be slightly higher with the LRP method than with the plasma method. In addition, for cases where only gram-positive bacteria were detected with the LRP method, the values were slightly higher than those obtained using the other two methods (Figure 3 ). ROC curves were created for the groups infected by gramnegative bacteria and other diseases (including gram-positive bacterial infection), and the sensitivity and specificity were calculated (Table 2). Groups in which gramnegative bacteria were detected in the blood culture were defined as true positive groups, whereas groups in which gram-positive bacteria or no infection were detected in the blood culture were identified as true negative groups. ROC curves were subsequently created between the two groups to calculate the sensitivity and specificity (Table 3 ). The LRP37 method showed the best diagnostic accuracy of the three measurement methods.
The sensitivity and specificity of techniques for diagnosing sepsis using conventional plasma endotoxin assays are too low, and plasma endotoxins are not detectable in many cases of gram-negative bacterial infection [1]. Kan et al. demonstrated that LRP is useful for the diagnosis of endotoxemia and found a clear improvement in the sensitivity and specificity of this assay compared with the plasma method $[1,6]$. Their experiments were performed with a commercially available HES solution, which is used as a cryopreservation agent for cultured cells. They incubated a mixture of the HES solution and blood at room temperature to obtain the supernatant (LRP). However, properties such as the molecular weight of the HES solution are not disclosed by the manufacturer. The production of HES was also discontinued recently. In addition, HES is less soluble in water, and preparing a saline solution can be difficult. Therefore, we used dextran instead of HES in this study.

The results indicated that dextran enhanced endotoxin activity, and that the enhancement was greater at $37^{\circ} \mathrm{C}$. Based on the study results, the most important question is how dextran increases the endotoxin activity. Endotoxins have a micellar structure and constitute gigantic associates of 100,000-1,000,000 $\mathrm{Da}$ [13]. We think that these gigantic associates moderately decrease in size in the presence of dextran. Thus, dextran may enter the micellar structure and break the endotoxin into a large number of smaller micellar struc-

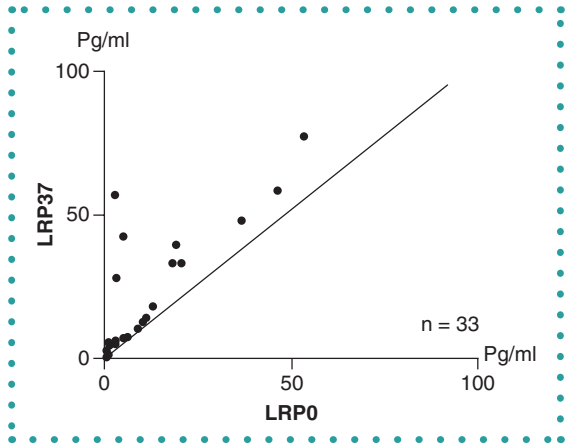

Figure 1. Measurement values of both methods in gram-negative bacteria detection examples. The measurement value of the LRP37 method was high in 27 of 33 cases.

LRP: Leukocyte-rich plasma.

tures. When the micelles increase in number, and convection occurs in an environment of $37^{\circ} \mathrm{C}$, it becomes easier for them to bind to the target molecule, that is, factor $\mathrm{C}$ in the LAL reagent. Furthermore, it is possible that the causative bacteria proliferated in the sample during culture at $37^{\circ} \mathrm{C}$ and the endotoxin levels may have increased. However, as illustrated in Figure 1, since the increase in the measured value was apparent even in the experiment using LPS, the reason for the increase in the measured value is unclear.

Yaegashi et al. [5] measured endotoxin levels in the white blood cell fraction (buffy coat) and reported the detection of endotoxins in this fraction, but not in the plasma, for some specimens. Kan et al. [1] reported that even if the endotoxin levels were below the detection limit for the plasma method, LRP endotoxin levels could be measured

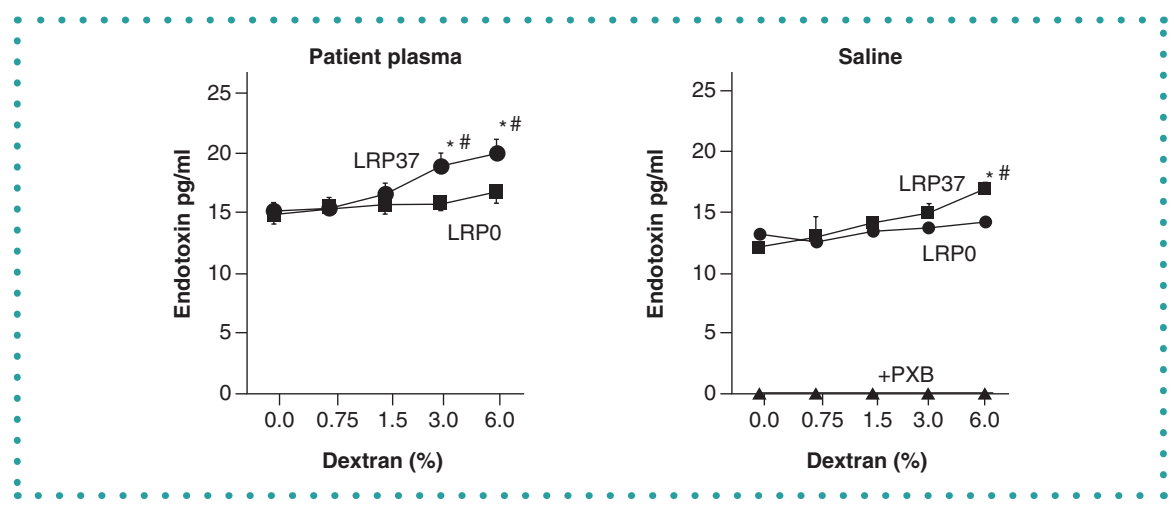

Figure 2. Effect of dextran concentration on the limulus activity of lipopolysacharide in physiological saline and endotoxemic patient plasma. LPS in physiological saline or endotoxemic patient plasma was incubated at 0 or $37^{\circ} \mathrm{C}$ in the presence of graded doses of dextran for $15 \mathrm{~min}$, followed by endotoxin measurement. PXB $(100 \mu \mathrm{g} / \mathrm{ml})$ was added to the LPS solution and the inhibitory effect was observed.

${ }^{*} \mathrm{p}<0.05$ (Student's t-test against 0 control).

$\# p<0.05$ (Student's t-test against the same dextran concentration).

LPS: Lipopolysaccharide; PXB: Polymyxin B. 


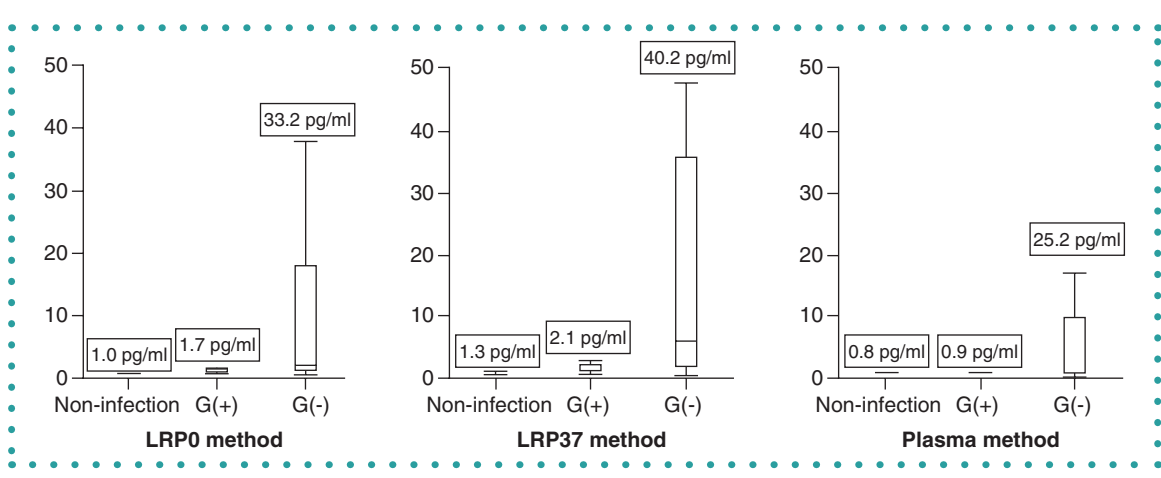

Figure 3. Patient endotoxin values with leukocyte-rich plasma and plasma methods. Box plots show the data for specimens obtained from patients with sepsis due to $\mathrm{G}(-)$ and $\mathrm{gG}(+)$ bacteria, and for noninfectious patients. The figures for the $\mathrm{G}(-)$ and $\mathrm{G}(+)$ groups represent the median values. G(-): Gram negative; G(+): Gram positive; LRP: Leukocyte-rich plasma.

in some specimens from patients with sepsis. Similar unexplainable results were also obtained in this study in approximately $8 \%$ of cases. Endotoxins positively bind to leukocytes through the endotoxin receptor to activate them, but not all endotoxins that enter into the circulation will immediately bind to leukocytes without first floating freely in the circulation. It may be that leukocytes capture endotoxins at the site of infection and subsequently enter into the circulation. Recently, a study demonstrated that although neutrophils are short-lived effector cells that undergo apoptosis in damaged tissues, in some settings they may leave the site of tissue injury and migrate back into the circulation [14]. Thus, the site at which endotoxins interact with leukocytes may also be the site of inflammation rather than in the circulation. Neutrophils are thought to play a major role in this context, but lymphocytes or platelets, in addition to monocytes, may also be carriers of endotoxins.

Even in cases of gram-positive bacterial infection, we could detect small concentrations of endotoxins around the cut-off value. In such cases, it is possible that gram-negative bacterial infections were hidden. However, if the patient has a serious condition, the strong bonds between the cells of intestinal villi may collapse, or villus cell destruction may occur, resulting in intestinal bacteria invading the site and leukocyte accumulation from blood vessels at the same site. Thereafter, leukocyte binding of endotoxins and reverse migration into the circulation may occur. As the presence of cell-surface markers for re-entered leukocytes has been reported [14], leukocyteassociated endotoxins may also become a valuable marker. A previous study demonstrated the existence of endotoxin-bound blood leukocytes in patients with Kawasaki disease [15]. There is a need to investigate methods for measuring leukocytebound endotoxins in the blood for various diseases with increased intestinal permeability, and the possibility that leukocytebound endotoxins are involved in their pathophysiology. Measuring leucocytebound endotoxins will not only improve diagnostic efficiency for sepsis, but it may also be a means of clarifying the role of endotoxins in diseases other than sepsis, which have only been estimated so far.

In conclusion, the important findings in this study are as follows:

- The LRP method using dextran T-500 does not require centrifugation, and samples can be produced at any facility;

- The LRP method using dextran T-500 has better measurement accuracy than the plasma method;

- The LRP37 method was superior to the LRP0 method in terms of isolation time and diagnostic accuracy.

The measurement sensitivity of the LRP37 method is expected to improve in the

Table 2. Measurement accuracy by receiver operating characteristic analysis.

\begin{tabular}{|l|l|ll|}
\hline & LRP37 method & LRP0 method & plasma0 method \\
\hline Area under the curve & 0.92 & 0.88 & 0.85 \\
\hline Cut-off value & 2.69 & 1.71 & 1.1 \\
\hline Sensitivity & 96.8 & 89.6 & 96.8 \\
\hline Specificity & 82.1 & 76.0 & 67.7 \\
\hline LRP: Leukocyte-rich plasma. & & \\
\hline
\end{tabular}

Table 3. Measurement accuracy by receiver operating characteristic analysis.

\begin{tabular}{|l|l|l|l|}
\hline & LRP37 method & \multicolumn{2}{l|}{ plasma0 method } \\
\hline Area under the curve & 0.99 & 0.97 & 0.76 \\
\hline Cut-off value & 0.97 & 1.07 & 0.66 \\
\hline Sensitivity & 96.8 & 90.9 & 100 \\
\hline Specificity & 100 & 95.0 & 53.4 \\
\hline LRP: Leukocyte-rich plasma. & &
\end{tabular}


future, and a simple method for endotoxin measurement, such as a point-of-care test, may be developed.

\section{FUTURE PERSPECTIVE}

At present, endotoxin has not been measured clinically because of the low accuracy of the assay. However, the accuracy was dramatically improved by the LRP37 method. Endotoxin is a very important causative agent of sepsis and is also important in the selection of antibiotics. The LRP37 method does not require special instruments and is expected to be widely used clinically. We expect that the standard for endotoxin measurement will change to this method in 5 years.

\section{AUTHOR CONTRIBUTIONS}

G Takahashi designed the study and wrote the initial draft of the manuscript. $K$ Inada, $\mathrm{K}$ Sato and $\mathrm{Y}$ Inoue contributed to the analysis and interpretation of data and assisted in the preparation of the manuscript. All other authors have contributed to data collection and interpretation, and critically reviewed the manuscript. All authors approved the final version of the manuscript, and agree to be accountable for all aspects of the work in ensuring that questions related to the accuracy or integrity of any part of the work are appropriately investigated and resolved.

\section{ACKNOWLEDGMENTS}

$S$ Endo and $\mathrm{K}$ Kishi advised us regarding the experimental methods used in this study. We would like to express the deepest appreciation to them

\section{FINANCIAL \& COMPETING INTERESTS DISCLOSURE}

The authors have no relevant affiliations or financial involvement with any organization or entity with a financial interest in or financial conflict with the subject matter or materials discussed in the manuscript. This includes employment, consultancies, honoraria, stock ownership or options, expert testimony, grants or patents received or pending, or royalties.

No writing assistance was utilized in the production of this manuscript.

\section{ETHICAL CONDUCT OF RESEARCH}

Ethical approval for the study (Ethical Committee no. H29-174) was provided by the Ethical Committee of Iwate Medical University, Morioka, Japan (Chairperson: Y Sato) on 15 March 2018. In addition, for investigations involving human subjects, informed consent has been obtained from the participants involved.

\section{OPEN ACCESS}

This work is licensed under the AttributionNonCommercial-NoDerivatives 4.0 Unported License. To view a copy of this license, visit http://creativecommons.org/licenses/ by-nc-nd/4.0/

\section{REFERENCES}

1. Kan $\mathrm{S}$, Takahashi G, Onodera $\mathrm{C}$ et al. Evaluation of an endotoxin-specific limulus amebocyte lysate assay using leukocyte-rich plasma for the diagnosis of gram-negative bacterial infection. J. Infect. Chemother. 19, 299-304 (2013).

2. Akira S, Takeda K. Toll-like receptor signaling. Nat. Rev. Immunol. 4, 499-511 (2004)

3. Vogel S, Hirschfeld MJ, Perera PY. Signal integration in lipopolysaccharide (LPS)-stimulated murine macrophages. J. Endotoxin. Res. 7, 237-241 (2001).

4. Lentschat A, El-Samalouti VT, Scheltter J et al. The internalization time course of a given lipopolysaccharide chemotype does not correspond to its activation kinetics in monocyte. Infect. Immun. 67, 2515-2521 (1999).

5. Yaegashi $Y$, Inada K, Sato N, Kojika M, Suzuki Y, Endo $\mathrm{S}$. [Endotoxin assay using blood leukocyte]. Endotoxin-Kenkyu 7, 182-188 (2004) (Annual report of Japan Endotoxin Society)

6. Kan S, Takahashi G, Onodera C et al. [Study on the leukocyte-rich plasma as the specimen for the measurement of endotoxin]. Rinshou. Byouri. 60, 1045-1052 (2012).

7. Strauss RG. In vitro comparison of the erythrocyte sedimenting properties of dextran, hydroxyethyl starch and a new low-molecular-weight hydroxyethyl starch. Vox. Sang. 37, 268-271 (1979).

8. Rubinstein P, Dobrila L, Rosenfield RE et al. Processing and cryopreservation of placental/umbilical cord blood for unrelated bone marrow reconstitution. Proc. Natl Acad. Sci. USA 92, 10119-10122 (1995).

9. Olofsson P, Olofsson C, Nylander G, Olsson P. Endotoxin inactivation in plasma from septic patients: an in vitro inactivation in plasma from septic patient
study. World J. Surg. 10, 318-323 (1986).

10. Oishi H, Takaoka A, Hatayama Y. Automated limulus amebocyte lysate (LAL) test for endotoxin assay using a new Toxinometer ET-201. J. Parenter. Sci. Technol. 39, 194-200 (1981).

11. Kambayashi JM, Yokota M, Sakon $M$ et al. A novel endotoxin-specific assay by turbidimetry with Limulus amebocyte lysate containing $\beta$-glucan. J. Biochem. Biophys. Methods 22, 93-100 (1991).

12. Inada $\mathrm{K}$, Endo $\mathrm{S}$, Takahashi $\mathrm{K}$ et al. Establishment of a new perchloric acid treatment method to allow detection of the total endotoxin content in human plasma by the Limulus test and clinical application. Microbiol. by the Limulus test and clinical
Immunol. 35, 303-314 (1991).

13. Gustsman T, Schromm AB, Brandenburg K. The physicochemistry of endotoxins in relation to bioactivity. Int. $\mathrm{J}$. Med. Microbiol. 297, 341-352 (2007).

14. de Oliveira S, Rosowski EE, Huttenloche A. Neutrophil migration in infection and wound repair: going forward in reverse. Nat. Rev. Immunol. 16, 378-391 (2016).

15. Takeshita S, Nakatani S, Kawase K et al. The role of bacterial lipopolysaccharide-bound neutrophils in the pathogenesis of Kawasaki disease. J. Infect. Dis. 179, 508-512 (1999). 